\title{
Encuadernadores zaragozanos del siglo XIX
}

\author{
Binders from Saragossa in the $19^{\text {th }}$ century
}

\author{
Lorena BAILO BENITO \\ Facultad de Ciencias de la Documentación de UCM, C/ Santísima Trinidad 37 28010, lobailo@ucm.es
}

\begin{abstract}
Resumen
Se presenta a los encuadernadores zaragozanos del siglo XIX que realizaron algunas de las encuadernaciones artísticas depositadas en la Biblioteca de la Universidad de Zaragoza. Para ello, primero se realizó un exhaustivo inventario de las encuadernaciones artísticas atesoradas en los depósitos de su Biblioteca General; luego se identificaron las encuadernaciones que presentaran firmas o etiquetas de encuadernadores zaragozanos; y finalmente se catalogaron las encuadernaciones con arreglo a la ficha normalizada del Catálogo Colectivo de Encuadernaciones Artísticas. Los resultados consisten en una breve descripción biográfica de los encuadernadores, la enumeración de las instituciones o particulares para los que trabajaron, y la realización de una somera descripción de los trabajos que realizaron para la Universidad de Zaragoza. Se concluye que los encuadernadores zaragozanos practicaron la mayoría de los estilos que estaban de moda en el resto de España, y que su oficio solía ser complementario la mayoría de las veces con el de impresor o el de librero.
\end{abstract}

Palabras clave: Encuadernación artística. Encuadernadores. Universidad de Zaragoza. Siglo XIX. Zaragoza.

\section{Introducción}

El siglo XIX español fue uno de los más convulsos de su historia, lleno de guerras, y de gobiernos liberales cambiantes e inestables. Durante la centuria, Zaragoza vivió los devastadores sitios de 1808 y 1809 , la victoria sobre los carlistas el 5 de marzo de 1838, así como un floreciente desarrollo urbanístico e industrial. Uno de los eventos más relevantes fue la Exposición Aragonesa de 1868, impulsada por la Real Sociedad Económica Aragonesa de Amigos del País, que pretendió ser un escaparate de la producción aragonesa y española de tamaño medio.

En lo relativo encuadernación, la industrial se impuso a la artesanal debido al aumento progresivo de las tiradas, de modo que la estructura constructiva se simplificó para producir un mayor número de encuadernaciones: el cosido se realizaba alla greca, y se usaba tarlatana como refuerzo del lomo y como medio de unión de los planos al lomo. En cuanto a los estilos

\begin{abstract}
The 19th century binders who made some artistic bookbindings which are kept in the Library of the University of Saragossa are presented. For that, firstly, an exhaustive inventory of the artistic bookbindings stored at its Historic Library warehouse was done. Then, those bookbindings with signatures or labels were identified. After that, bookbindings were catalogued following the Catálogo Colectivo de Encuadernaciones Artísticas cataloguing standard. The results consist of a brief biographical description of the binders, an enumeration of the institutions or people they worked with, and a brief description of the works they performed for the University of Saragossa. It can be concluded that binders from Saragossa practised most of the binding styles that then were fashionable in Spain. Moreover, binders used to be also printers or booksellers; thus, binding was a secondary occupation.
\end{abstract}

Keywords: Artistic bookbinding. Binders. University of Saragossa. $19^{\text {th }}$ century. Saragossa.

decorativos, a lo largo del siglo XIX predominaron cinco: neoclásico, imperio, de cortina, romántico, dividido en dos vertientes: a la catedral y romántica-isabelina, y técnico-industrial.

Este trabajo forma parte de la de tesis doctoral que se está llevando a cabo actualmente sobre las encuadernaciones artísticas de la Biblioteca General de la Universidad de Zaragoza. Para poder trabajar sobre el objeto de estudio, se firmó un convenio entre esta y el Grupo Bibliopegia, perteneciente a la Universidad Complutense de Madrid.

\section{Objetivos y metodología}

El objetivo del presente artículo son los siguientes: dar a conocer a los encuadernadores encontrados y elaborar una breve descripción de cada una de las encuadernaciones halladas, acompañada de fotografías. 
Para llegar a este objetivo, en primer lugar se realizó un inventario exhaustivo de las encuadernaciones artísticas atesoradas en los depósitos de la Biblioteca General, estableciendo una acotación temporal que abarcaba desde las más antiguas, es decir del siglo XV, hasta 1900. Después, se identificaron las encuadernaciones entre las encuadernaciones del siglo XIX, se encontraron las firmas y las etiquetas de algunos encuadernadores zaragozanos: Francisco Magallón, C. Muñoz, Emilio Fortún y Comas Hermanos. Posteriormente se catalogaron las encuadernaciones de estos artesanos según la ficha establecida en el Catálogo Colectivo de Encuadernaciones Artísticas, analizando las técnicas constructivas, los utensilios y estructuras decorativos y los aspectos documentales de cada una de las encuadernaciones descritas en este artículo.

\section{Encuadernadores zaragozanos}

\subsection{Francisco Magallón}

Francisco Magallón fue impresor (Borao, 1995: 71) y encuadernador (López Serrano, 1972, 77; Checa Cremades, 2003: 379; Carpallo Bautista, 2015: 34; Massó Valdés, 2015: 246) entre 1789 y 1831. Como encuadernador, cultivó dos estilos: el neoclásico y el de cortina. Su sucesor fue Eusebio Magallón (Borao, 1995: 85; Flores Hernández, 2016: 1440), del cual solamente se conoce que cultivó el estilo romántico isabelino.

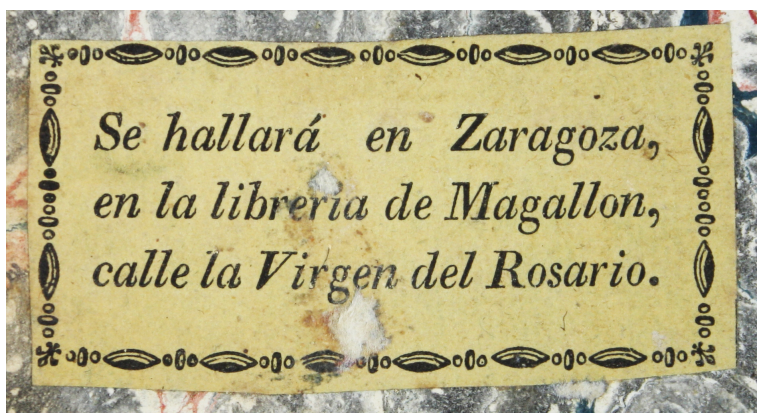

Figura 1. Etiqueta de Francisco Magallón

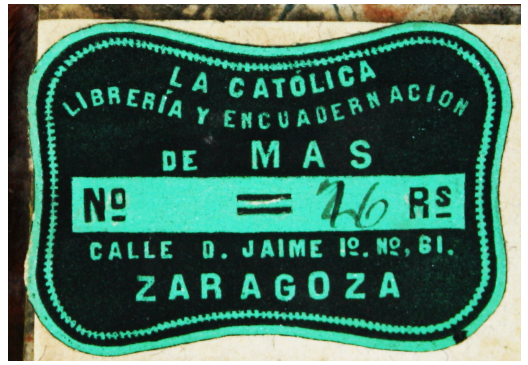

Figura 2. Etiqueta de La Católica Librería y Encuadernación
La Biblioteca Universidad de Zaragoza conserva dos encuadernaciones de estilo neoclásico de Francisco Magallón, las cuales recubren los dos volúmenes de las Obras de Cayo Salustio Crispo, traducidas por el Infante Gabriel. Madrid: [s.n.], 1804 (Imprenta Real). (Signaturas G-77-136 y G77-137). La obra presenta dos etiquetas pegadas en la guarda anterior del primer volumen: la primera menciona la librería de Magallón en la calle Virgen del Rosario (Figura 1), a la cual el artesano se trasladó en 1814 (Borao, 1995: 71); la segunda señala una "Librería Católica" (Figura 2) situada en la Calle don Jaime, la cual pudo ser la regentada por el impresor José Bedera situada también en Virgen del Rosario, es decir, en el tramo de la calle Don Jaime I situado entre las calles Mayor y Espoz y Mina y la plaza Ariño (Moneva y Puyol 1949: 93-102; Blasco Ibáñez, 2018), de modo que un sucesor de Magallón pudo haber traspasado el negocio a Bedera.

En todo caso, la estructura decorativa de los planos es simétrica, que consiste en una pasta española sobre la cual se han estampado y dorado dos encuadramientos, realizados con una rueda de motivos vegetales (Figura 3).

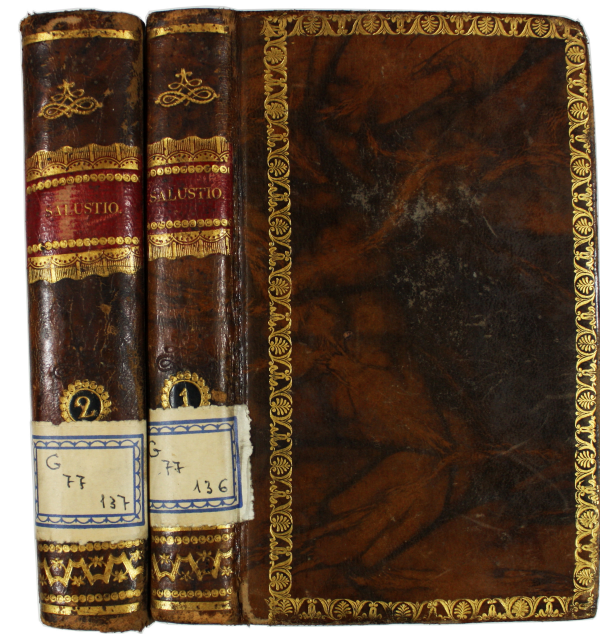

Figura 3. Vista general de los lomos $y$ del plano anterior de G-77-136 y G-77-137

El lomo presenta mayor complejidad: dos tejuelos con el autor estampado en letras doradas, otros dos redondos y negros con los números de parte, florones dorados y gofrados y cuatro tipos de paletas: lisas, de puntos, semicírculos punteados rematados con líneas verticales paralelas, y un zig-zag rematado por florecillas (Figura 3).

La decoración de las encuadernaciones se completa en las guardas, presentan un marmoleado tipo shell (Figura 4), y en los cortes coloreados y salpicados. 


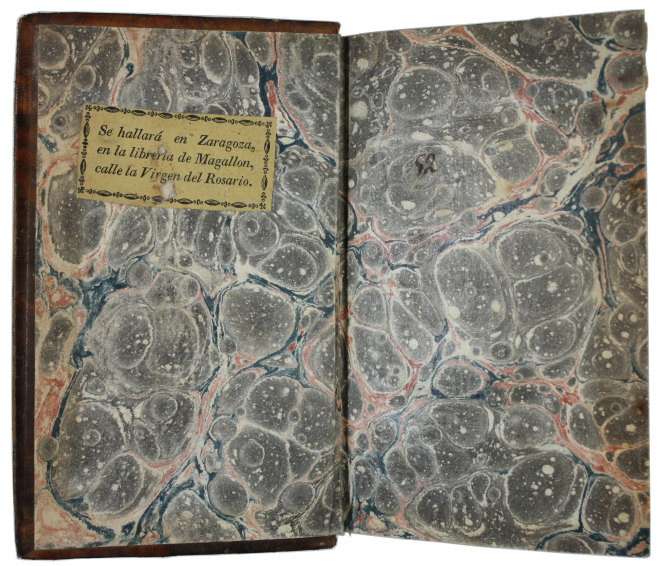

Figura 4. Guarda anterior con marmoleado tipo shell de G-77-136

\subsection{Muñoz}

De este encuadernador solamente se conoce su nombre y su profesión a través de su firma en letras doradas en el lomo "C. MUÑOZ" (Figura 5), y también por una etiqueta encolada en la guarda anterior "MUÑOZ ENCUADERNADOR" (Figura 6). Por el momento no es conocida la ciudad en la que trabajaba este encuadernador, puesto que esta no se ha encontrado ni en la inscripción de la cofia ni en la etiqueta del interior. Como no se ha localizado más información del encuadernador en fuentes bibliográficas, y la tesis está aún en fase de análisis documental de las encuadernaciones, pueden establecerse dos hipótesis sobre la ciudad donde operaba el artesano: en Zaragoza, ya que la encuadernación se ha localizado en los fondos de la Universidad de Zaragoza; o bien en Madrid debido a que las obras que contiene el libro se imprimieron en esta ciudad.

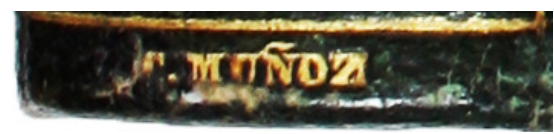

Figura 5. Firma del encuadernador en la cofia inferior

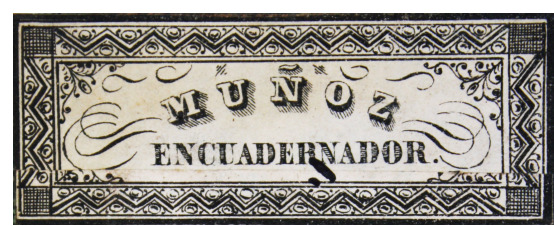

Figura 6. Etiqueta de C. Muñoz en la guarda anterior

La Biblioteca de la Universidad de Zaragoza conserva una encuadernación de Muñoz de estilo romántico isabelino que cubre un volumen facticio que contiene dos obras: la Constitución de la monarquía española promulgada en Madrid a 18 de julio de 1837...Madrid, [s.n.], 1837 (Imprenta Nacional), y la Colección de constituciones...Madrid, [s.n.], 1836 (Imprenta del Eco del Comercio) (Signatura H-14-44).

Con respecto a la decoración, esta se concentra principalmente en la cubierta, cuya estructura decorativa es simétrica, que consiste en una piel de color verde botella con una alta saturación de motivos en oro: cuatro hilos exteriores y un motivo central, compuesto de varias planchas de hojas de acanto, veneras y jarrones (Figura 7).

El lomo presenta una disposición más sencilla, también en dorado: consta de cuatro grecas flanqueadas por dos filetes, dos junto a las cofias y las otras dos en el centro. Estas dos últimas, junto a dos filetes curvos, enmarcan el título del volumen facticio "COLECCIÓN de CONSTITUCIONES" en letras doradas. La composición entera está enmarcada por dos filetes verticales. En la cofia inferior, como se ha dicho con anterioridad, se localiza la firma del encuadernador en letras doradas "C. MUÑOZ" (Figura 7).

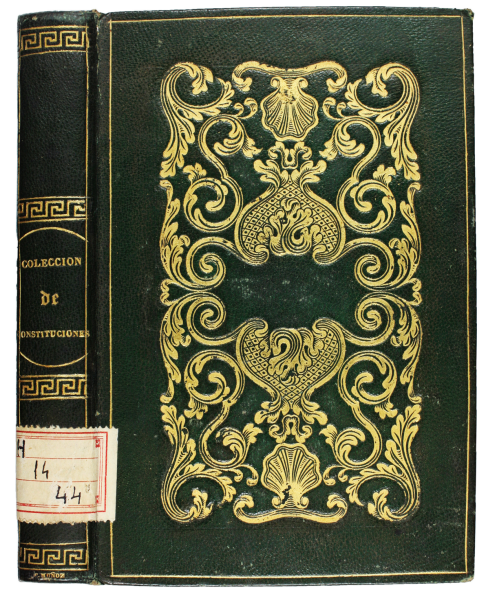

Figura 7. Vista general del plano anterior y del lomo

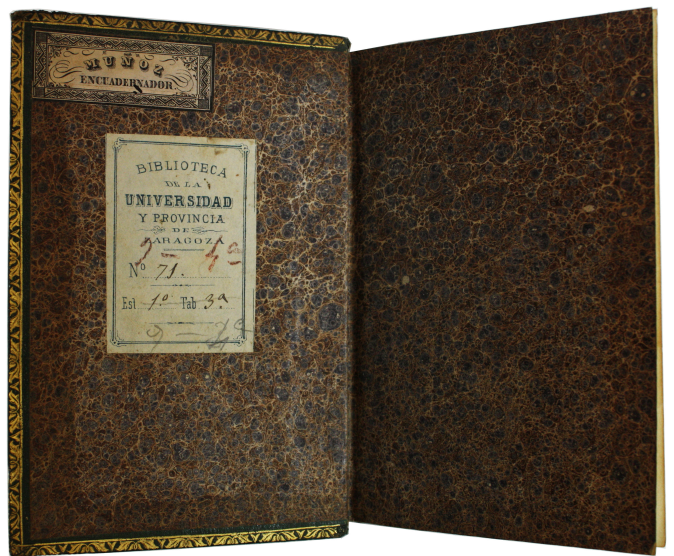

Figura 8. Guarda anterior con marmoleado tipo shell 
La decoración de la encuadernación se completa con grecas doradas en las cofias y las esquinas y una rueda vegetal en las vueltas de la piel. Asimismo, las hojas de guarda presentan un marmoleado tipo shell (Figura 8), y los cortes se han salpicado.

\subsection{Emilio Fortún}

Emilio Fortún ejerció de encuadernador en el último cuarto del siglo XIX. Firmaba sus encuadernaciones mediante una etiqueta colocada en la contratapa anterior "ENCUADERNACIÓN Y RAYADOS EMILIO FORTÚN CINCO DE MARZO 2 ZARAGOZA" (Figura 9). Trabajó para diversas instituciones españolas y aragonesas como el Hospicio Provincial, la Maestranza Zaragozana (López Serrano, 1972: 91), el Casino Mercantil de Zaragoza (Moralejo y Pedraza, 1983: 122), la Biblioteca de Palacio o la Diputación de Zaragoza, que regaló dos libros encuadernados por este artífice a Amadeo de Saboya con motivo de su visita a la ciudad. Fortún también trabajó para particulares como el fotógrafo oscense Félix Preciado (Hernández Latas, 2015: 120). Asimismo, el artesano participó en la Exposición Aragonesa de 1868 y fue premiado con una medalla de cobre en la categoría de artes liberales.

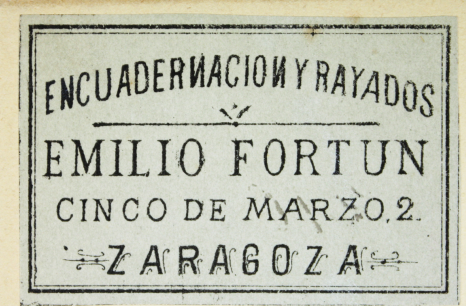

Figura 9. Etiqueta de Emilio Fortún
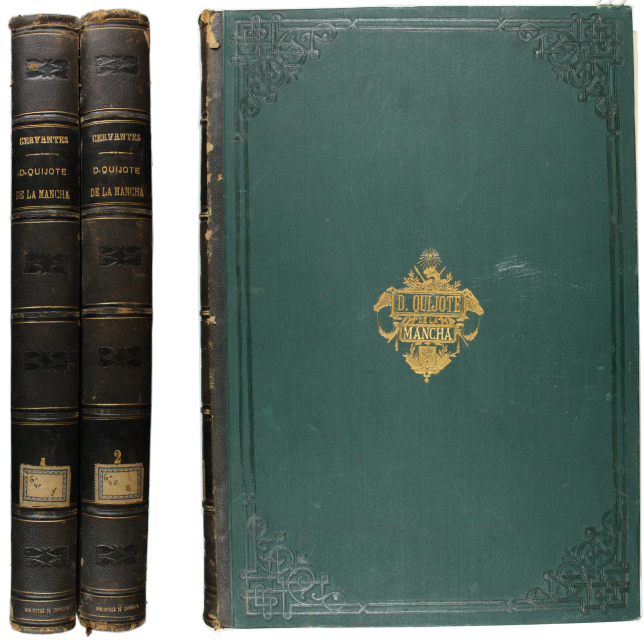

Figura 10. Vista general de los lomos y del plano anterior de G-40-7 y G-40-8
Fortún fue, con diferencia, el artífice que más libros encuadernó para la Biblioteca de la Universidad de Zaragoza: se localizaron 84 ejemplares, la mayoría de ellos recubiertos con encuadernaciones a la holandesa, pero seis ostentan una encuadernación artística de estilo industrial: concretamente, la primera y segunda parte de El Quijote de Cervantes (Barcelona: Tomás Gorchs, 1859) (Signaturas G-40-7 y G-40-8) (Figura 10), los tres volúmenes de Viaje a Oriente de la fragata de guerra Arapiles... de Juan de Dios de la Rada y Delgado (Barcelona: Emilio Oliver y Compañía, 1878) (Signaturas H-14-6, H-14-7 y H-14-8) (Figura 11), y el primer volumen de Historia de las órdenes de caballería y de las condecoraciones españolas (Madrid: [s.n.], 1864 (en la Imprenta de Tomás Rey)) (Signatura H-14-11) (Figura 12).
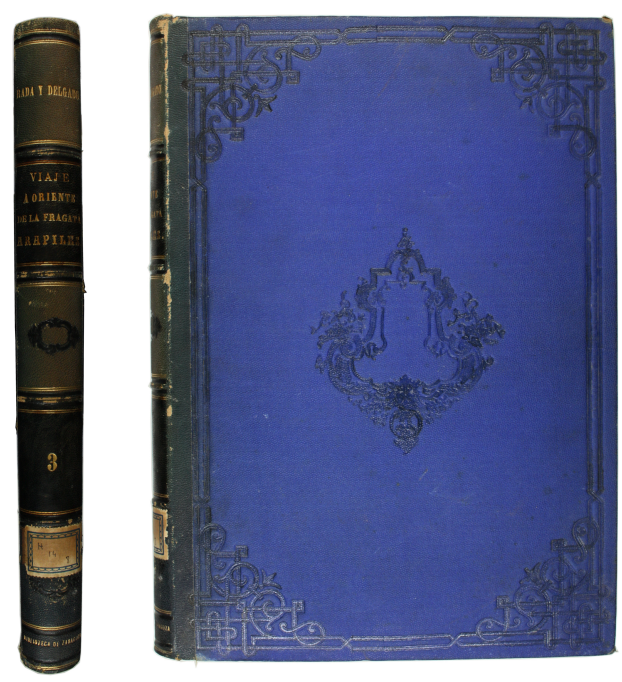

Figura 11. Vista general del plano anterior y del lomo de $\mathrm{H}-14-8$
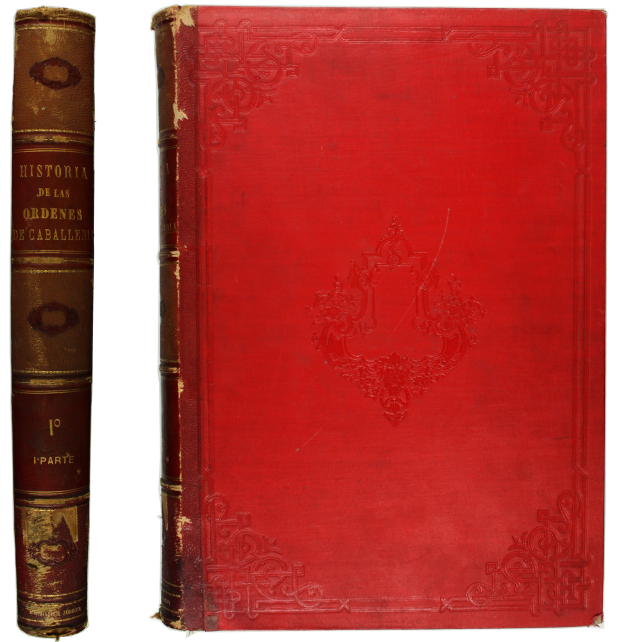

Figura 12. Vista general del plano anterior y del lomo de $\mathrm{H}-14-11$ 
Todas las encuadernaciones de este último tipo comparten características comunes: Todas son encuadernaciones a la holandesa con piel en el lomo y tela en los planos -elementos verdes en Don Quijote, azules en Viaje a Oriente... y rojos en Historia de las órdenes de caballería...siendo simétrica la estructura decorativa de los planos, compuesta de cuatro planchas geométricas en las esquinas, realizadas en tres hilos, el central más ancho que los extremos, las cuales combinan elementos rectos, roleos y un pequeño motivo vegetal. Las planchas están unidas mediante dos conjuntos de tres filetes. Los lomos también son similares, pues todos presentan pequeñas planchas geométricas, rotulado en dorado y el super libros "BIBLIOTECA DE ZARAGOZA" (Figura 13) junto a la cofia inferior.

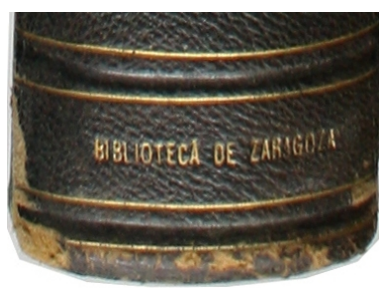

Figura 13. Super libros de la Biblioteca de la Universidad de Zaragoza

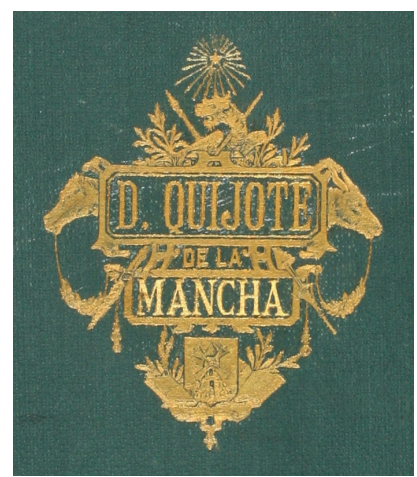

Figura 14. Plancha central, utilizada en los planos de G-40-7 y G-40-8, en la que se puede observar la iconografía de Don Quijote de la Mancha

Los motivos centrales, sin embargo, muestran algunas diferencias. En Viaje a Oriente... y en Historia de las órdenes de caballería... las planchas están formadas por elementos geométricos y vegetales, mientras que Don Quijote presenta una plancha dorada con el título abreviado y en mayúsculas encerrado en tres cartelas, alrededor de las cuales se ha dispuesto la iconografía de la novela: una cabeza de dragón, Rocinante, el rucio, una espada y una lanza entrecruzadas, libros y un molino manchego (Figura 14).

Don Quijote e Historia de las órdenes de caballería... contienen papel de guardas impreso con motivos de inspiración oriental, acorde con la moda europea de la época, los cuales consisten en cuatro pequeñas escenas campestres de humanos y animales entre pagodas y motivos vegetales. Las cuatro escenas se alternan en dos hileras (Figuras 15 y 16).

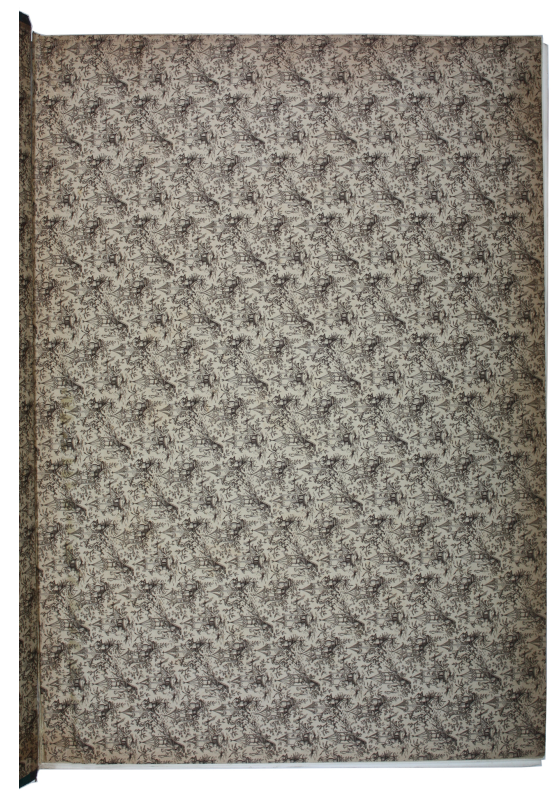

Figura 15. Vista general de la guarda impresa de motivos orientales utilizada en G-40-7, en G-40-8 y en $\mathrm{H}-14-11$

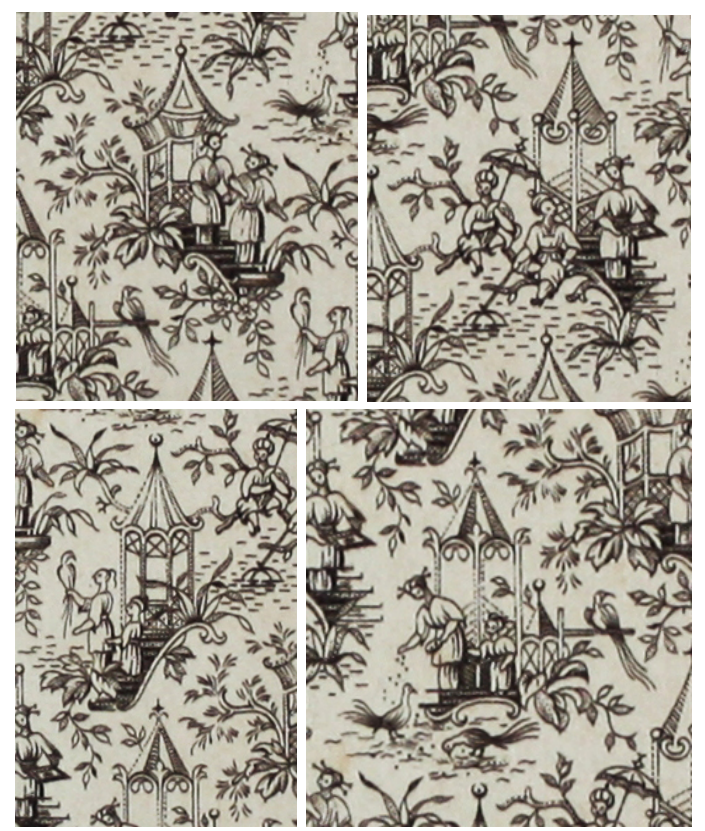

Figura 16. Detalles de las cuatro escenas que se pueden observar en las hojas de guardas utilizadas en G-40-7, en G-40-8 y en $\mathrm{H}-14-11$

De izquierda a derecha. dos mujeres hablando, una mujer llevando una bandeja y dos hombre tomando el sol, un padre y un hijo practicando cetrería

y una madre y una hija dando de comer a las aves 


\subsection{Comas Hermanos}

Juan y Mariano Comas Barba ejercieron de impresores, libreros y encuadernadores. Su establecimiento estaba situado en dos locales, Plaza del Pilar 40 y Paseo del Ebro 50 (Blasco Ibáñez, 2018). Realizaron algunos trabajos para la Biblioteca de Palacio (López Serrano, 1972: 91) y pudieron trabajar junto a su padre en encuadernaciones para el Casino Mercantil de Zaragoza, firmando como "A. Comas e Hijos" (Moralejo y Pedraza, 1983: 122).

La Biblioteca Universidad de Zaragoza conserva dos encuadernaciones de Comas Hermanos, ambas de estilo industrial: la primera obra, Crónica del segundo Congreso Nacional Español: discursos... Zaragoza, [s.n.], 1891 (Mariano Salas) (Signatura G-14-48), presenta una cubierta en tela roja sobre la cual se han aplicado las técnicas del dorado y del entintado (Figuras 17 y 18).

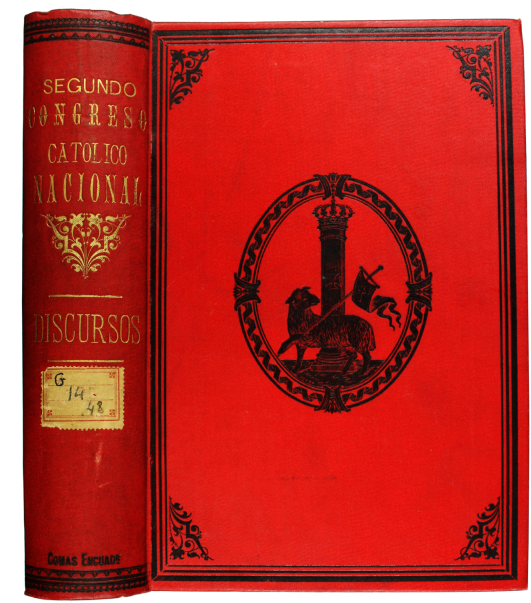

Figura 17. Vista general del plano anterior y del lomo de G-14-48

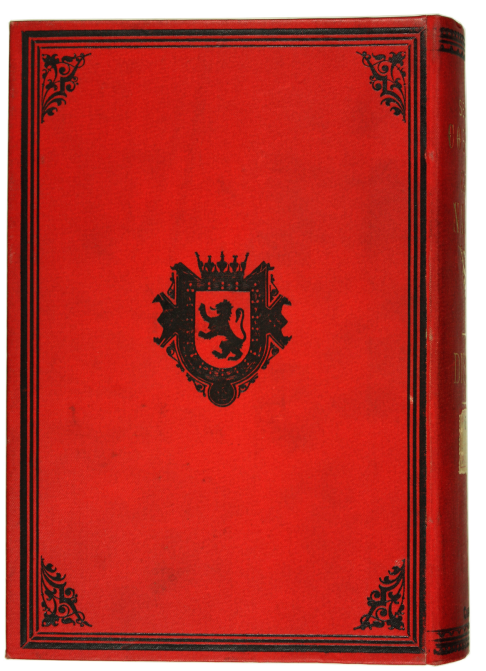

Figura 18. Vista general del plano posterior de G-14-48
La estructura decorativa es asimétrica: ambos planos constan de encuadramientos realizado con tres filetes, rematados con motivos vegetales, mientras las planchas centrales son distintas: la del plano anterior es una plancha ovalada que contiene una columna coronada, símbolo de la Virgen del Pilar, custodiada por un agnus dei (Figura 17); mientras que la del plano posterior representa el escudo de Zaragoza coronado, el cual contiene el león rampante símbolo de la ciudad (Figura 18).

El lomo presenta la siguiente composición: dos paletas geométricas y el título y subtítulo en letras doradas, con un florón entre ellos y flanqueado por dos filetes (Figura 17). La firma del encuadernador "COMAS ENCUAD" (Figura 19) se localiza junto a la cofia inferior.

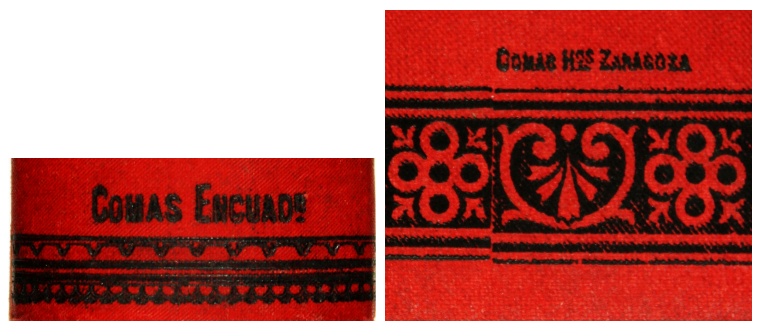

Figura 19. Firmas del encuadernador en G-14-48 y G-14-54

La segunda obra, Tratado elemental de Física experimental y aplicada para uso de las universidades, escuelas especiales, etc. escrito por Bartolomé Feliú y Pérez e impreso en Zaragoza, en la Tipografía de Comas Hermanos, 1896 (Signatura G-14-54), presenta una cubierta de tela roja sobre la cual se ha aplicado las técnicas del entintado (Figuras 20 y 21).

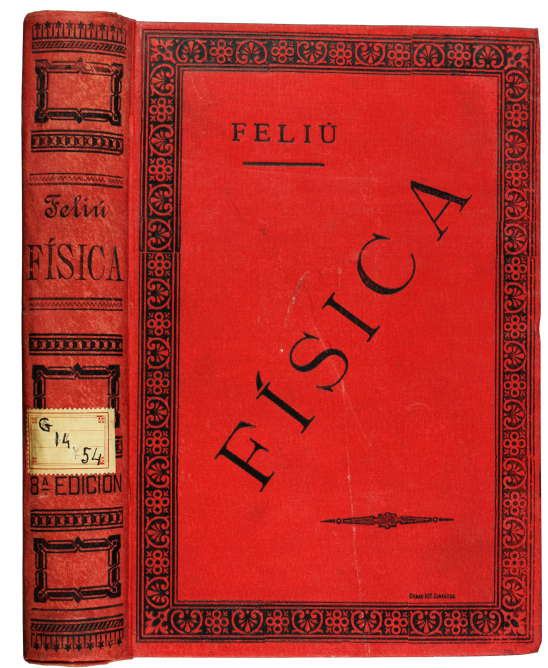

Figura 20. Vista general del plano anterior y del lomo de G-14-54 
La estructura decorativa de los planos es asimétrica: el plano anterior consiste en un encuadramiento exterior formado por cuatro planchas esquineras y seis planchas rectas de motivos vegetales esquematizados. El interior manifiesta una disposición en Z: el título "FíSICA" es el trazo inclinado y el autor "FELIÚ" y la pequeña plancha geométrica constituyen, respectivamente, los trazos superior e inferior (Figura 20). La firma "COMAS $H^{\text {NOS }}$ ZARAGOZA" se localiza en la esquina inferior derecha (Figura 19). La composición del plano posterior consta de una plancha ovalada en el centro rematada por cuatro florones (Figura 21).

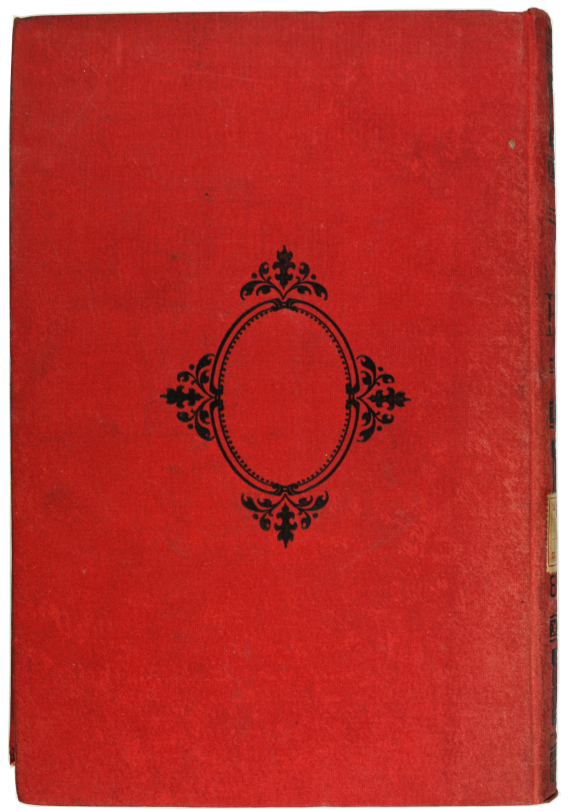

Figura 21. Vista general del plano posterior de G-14-54

La composición del lomo, más compleja que la de los planos, está formada por varias planchas rectangulares formadas por el entrecruzamiento de tres hilos, y varios tipos de paletas: una de aspecto cordiforme flanqueada por dos filetes, el inferior rematado por líneas verticales y estrellas; un doble filete que contiene una línea en zig-zag, formando el autor "Feliú" y el título "FÍSICA" un falso tejuelo enmarcado por dos paletas de este tipo; y un doble filete que contiene hexágonos con la mitad entintada y la otra mitad no, la indicación de edición "8A EDICIÓN" se localiza entre dos paletas de este tipo (Figura 20).

\subsection{Conclusiones}

Dar a conocer a los encuadernadores zaragozanos del siglo XIX y sus trabajos nos permite observar que el oficio de encuadernador solía ser secundario y que estos artesanos trabajaban principalmente de impresores o de libreros:
Francisco Magallón fue conocido principalmente por su labor de impresor, y los Hermanos Comas se dedicaron principalmente a la impresión y a la venta de libros. Sin embargo, Emilio Fortún fue el único profesional de los mencionados en este artículo que se dedicó en exclusiva a la manufactura de encuadernaciones. De C. Muñoz no ha sido posible averiguar si trabajaba exclusivamente de encuadernador o si se dedicó también a ser librero o impresor.

Se puede apreciar también que el oficio de encuadernador seguía estando activo en Zaragoza durante todo el siglo XIX, donde permearon la mayoría de los estilos artísticos explicados en la introducción, además de que la mayoría de estos encuadernadores trabajaron además para otras instituciones y particulares aragoneses, y realizaron también encuadernaciones de lujo para la Biblioteca de Palacio, como es el caso de Emilio Fortún y de Comas Hermanos, explicado más arriba, aunque sus aportaciones a la colección de encuadernaciones no han recibido el mismo reconocimiento que las de otros encuadernadores más afamados — como los madrileños Antonio de Sancha y Gabriel de Sancha, padre e hijo respectivamente, el valenciano Pascual Carsí y Vidal o los catalanes Pedro Doménech y Hermenegildo Miralles- Como muestra de ello, las encuadernaciones de estos últimos encuadernadores se han catalogado en la Base de Datos de Encuadernación Histórico-Artística elaborada por la Biblioteca de Palacio, mientras que las de Emilio Fortún simplemente presentan una descripción somera en el catálogo bibliográfico de la biblioteca, concretamente en las notas de ejemplar de los libros que contienen, y las de Comas Hermanos ni tan siquiera consta en ninguno de los dos catálogos.

Las encuadernaciones objetos de la tesis doctoral están actualmente en proceso de análisis documental, de forma que se necesitaría complementar el conocimiento que ha proporcionado las fuentes bibliográficas con la consulta de fuentes primarias. Para ello se consultarían los archivos parroquiales de Zaragoza para comprobar las partidas de bautismo, de matrimonio o defunción de los encuadernadores, con el fin de poder arrojar más datos tanto biográficos como profesionales de los mismos.

\section{Notas}

(1) Los derechos de las fotografías son de la Universidad de Zaragoza y de Antonio Carpallo Bautista.

\section{Referencias}

Base de Datos de Encuadernación Histórico-Artística (2020) [Madrid]: Real Biblioteca, [s.a.]. https://encuadernacion.realbiblioteca.es/ (2020-06-16). 
Biblioteca Virtual de Aragón (2020). Zaragoza: Gobierno de Aragón; Madrid: Ministerio de Cultura y Deporte. Secretaría de Estado de Cultura, [s.a.]. http://bibliotecavirtual. aragon.es/bva/i18n/estaticos/contenido.cmd?pagina=estaticos/presentacion (2020-03-08).

Blasco Ibáñez, Fátima (2018). Los impresores zaragozanos a comienzos del siglo XX: un sector en transición. // Revista Digital de la Asociación Aragonesa de Críticos de Arte. ISSN 1988-5180. N N 44 (2018). http://www.aacadigital.com/contenido.php?idarticulo=1449 (2020-06-15).

Borao, Gerónimo (1995). La imprenta en Zaragoza. Ed. facs. [Zaragoza]: Caja de Ahorros y Monte de Oiedad de Zaragoza, Aragón y Rioja, 1995. ISBN 84-88793-40-5.

Carpallo Bautista, Antonio (2015). Encuadernaciones del siglo XIX en la Biblioteca Histórica Municipal de Madrid. Madrid: Ollero y Ramos, 2015. ISBN 8478952946.

Checa Cremades, José Luis (2003). Los estilos de encuadernación: Siglo III d. J. C. - siglo XIX. Madrid: Ollero y Ramos, 2003. ISBN 84-7895-181-4.

Exposición Aragonesa de 1868 (2020). Catálogo de los expositores premiados a propuesta de la Junta General del Jurado Zaragoza: [s. n.], [ca. 1868]. 83 pp. http://bibliotecavirtual.aragon.es/bva/i18n/catalogo_imagenes/grupo.c md?path=3706347. $(2020-03-23)$

Exposición Aragonesa de 1868 (2020). Gran Enciclopedia Aragonesa. Zaragoza: DiCom, [s.a.]. http://www.enciclopedia-aragonesa.com/voz.asp?voz_id=5396\&tipo_busqu eda $=1 \&$ nombre $=$ Exposici\%F3n\%20aragonesa\%201868\&categoria id=\&subcategoria id=\&conlmagenes $=(2020-03-06)$.

Flores Hernández, Yohana Yessica; Carpallo Bautista, Antonio (dir.). (2016). Estudio, catalogación y digitalización de las encuadernaciones artísticas de la Real Academia de Bellas Artes de San Fernando. Madrid: Universidad Complutense de Madrid. Facultad de Ciencias de la Documentación, 2016. Tesis Doctoral

Hemeroteca Digital (2019). Madrid: Biblioteca Nacional de España, 2019. http://www.bne.es/es/Catalogos/HemerotecaDigital/ (2020-03-08)
Hernández Latas, José Antonio (2015). Fotógrafos y viajeros en torno al Balneario de Panticosa (Huesca): de Charles Clifford (1859) a Lucas Cepero (1915). // Argensola. Revista de Ciencias Sociales del Instituto de Estudios Altoaragoneses [en línea]. ISSN 0518-4088. № 125, (2015) 89131. http://revistas.iea.es/index.php/ARG/article/viewFile /2607/2599 (2020-03-23)

López Serrano, Matilde (1972). La encuadernación española: breve historia. Madrid: Asociación Nacional de Bibliotecarios, Archiveros y Arqueólogos, 1972.

Massó Valdés, Juan Bautista; Carpallo Bautista, Antonio (dir.) (2015). Análisis documental de las encuadernaciones artísticas del siglo XIX de la Biblioteca de la Real Academia de la Historia. Madrid: Universidad Complutense de Madrid. Facultad de Ciencias de la Documentación, 2015. Tesis Doctoral

Moralejo Álvarez, María Remedios; Pedraza Prades, María Dolores (1983). La Biblioteca del Casino de Zaragoza. // Cuadernos de Aragón. ISSN 0590-1626.№ 16-17(1983), 113-126. https://ifc.dpz.es/recursos/publicaciones/11/78/ 02moralejopedraza.pdf ( 2020-03-23)

Moneva y Puyol, Juan (1949). Josef Bedera: Impresor y librero católico. En Comerciantes de altura. Zaragoza: Librería General, 1949, pp. 93-102.

Proyecto de Censos y Padrones en Aragón. Siglo XIX y comienzos del XX (2012). [S.I.]: Asociación Cultural de Genealogía e Historia de Aragón, 2012 - http://www.aragongen.org/public/index.php (2020-03-24).

Zaragoza. Gran Enciclopedia Aragonesa (2020). Zaragoza: DiCom, [s.a.] http://www.enciclopedia-aragonesa.com/ voz.asp?voz_id=13418\&tipo_busqueda=1\&nombre $=\mathrm{Za}$ ragoza\&categoria_id=4\&subcategoria_id=21\&conlmagenes=\#Punto_7 (2020-03-07).

Enviado: 2020-05-06. Segunda versión: 2020-06-17. Aceptado: 2020-06-23. 